\section{Prevenção da deficiência mental: conhecimento e percepção dos profissionais de saúde}

\author{
Prevention of mental retardation: knowledge \\ and perception by health professionals
}

\author{
1 Centro de Ciências \\ da Saúde, Universidade \\ Estadual de Maringá, \\ Maringá, Brasil. \\ 2 Faculdade de Ciências \\ Médicas, Universidade \\ Estadual de Campinas, \\ Campinas, Brasil. \\ Correspondência \\ A. M. S. Machado \\ Departamento de Medicina \\ Centro de Ciências da Saúde, \\ Universidade Estadual \\ de Maringá. \\ Av. Mandacaru 1590 \\ Maringá, $P R$ \\ 87083-240, Brasil. \\ amsmmoraes@uem.br
}

\section{Abstract}

This article aimed to evaluate the prevention of mental disability in primary healthcare services in Maringá, Paraná, Brazil. The sample consisted of 90 male and female physicians from different fields, namely gynecology and obstetrics, pediatrics, general practice, and family health, as well as 66 male and female nurses. A multiple-choice questionnaire was filled out by the subjects themselves from August to December 2003. Qualitative variables were compared using the qui-square test at 5\% significance level. Partial data relating to both the perception and knowledge of health professionals concerning mental disability were as follows: $75 \%$ were unable to choose the correct alternative on prevalence; $25 \%$ did not know how the genome contributes to etiology; $37 \%$ were unaware of prevention for mental disability; $28 \%$ were not confident in providing orientation on the teratogenic effect of ethanol; 35\% demonstrated insecurity in orienting patients on amniocentesis. The data showed that participants had an unsatisfactory perception of the relevance of mental disability within the overall population disease profile, and that they need more information on the respective genetic and environmental issues.

Mental Health; Health Services; Perception
Ana Maria Silveira Machado de Moraes 1 Luís Alberto Magna 2 Antonia Paula Marques-de-Faria ${ }^{2}$

\section{Introdução}

A deficiência mental afeta entre 2 e $3 \%$ da população geral e $1 \%$ das crianças em idade escolar 1,2. No Brasil, 1,6\% da população apresenta essa condição 3 , valor provavelmente subestimado, mas suficiente para ser considerada como problema de saúde pública 4 .

O objetivo da pesquisa foi traçar o perfil do atendimento preventivo em deficiência mental na rede básica de saúde do Município de Maringá, Paraná, Brasil. Neste artigo são apresentados e discutidos dados parciais relacionados à percepção e ao conhecimento de profissionais de saúde sobre o tema.

\section{Casuística e método}

Trabalho desenvolvido entre médicos e enfermeiros que atuam nas Unidades Básicas de Saúde de Maringá. Estudo observacional, descritivo e transversal, realizado entre agosto e dezembro de 2003.

A amostra foi composta por 90 médicos(as) e 66 enfermeiros(as). Foram eleitos para a pesquisa médicos(as) com atuação em uma ou mais das seguintes áreas: Ginecologia e Obstetrícia, Pediatria, Clínica Geral, Programa Saúde da Família (PSF); bem como enfermeiros(as), que atuam na rede de saúde pública do município em estudo. Foram excluídos os profissio- 
nais em função administrativa, atuando em ambulatórios de especialidades e os que se recusaram a participar da pesquisa.

Foi aplicado questionário, por autopreenchimento, contendo 26 questões com respostas de múltipla escolha, com as alternativas "sim", "não" e "não sei" ou "não lembro", segundo método proposto por Gil 5 .

Os profissionais de saúde foram contactados pessoalmente e individualmente no local de trabalho. Aceitando participar, era solicitada a assinatura do Termo de Consentimento Livre e Esclarecido e entregue o questionário para ser recolhido após dois ou três dias.

O projeto foi aprovado pelo Comitê Permanente de Ética em Pesquisa Envolvendo Seres Humanos, da Universidade Estadual de Maringá, e sua execução no âmbito da rede pública, autorizada pela Secretaria Municipal de Saúde.

Para criar o banco de dados e fazer a análise estatística foi utilizado o programa SPSS 8.0 (SPSS Inc., Chicago, Estados Unidos). As variáveis qualitativas foram comparadas pelo teste do qui-quadrado, com nível de significância de 5\%.

\section{Resultados}

Os profissionais de saúde participantes e algumas características estão descritas nas Tabelas 1 e 2 .

Para 25\%, a prevalência da deficiência mental estaria entre 2 e $3 \%$; para outros $25 \%$, abaixo de $1 \%$ e outros $25 \%$ referiram não saber, sendo esta última resposta mais freqüente entre os enfermeiros $\left(\chi^{2}=14,874 ; p=0,011\right)$. O genoma contribui com até $10 \%$ das causas para $29 \%$ dos respondentes; para 34\%, a contribuição está entre 20 e $50 \%$ e ainda $25 \%$ disseram não saber, sendo este último grupo mais prevalente entre os enfermeiros $\left(\chi^{2}=11,203 ; p=0,024\right)$. Não sabiam se a deficiência mental pode ser prevenida

Tabela 1

Profissionais de saúde participantes da pesquisa.

\begin{tabular}{lrr}
\hline & $\mathbf{n}$ & $\%$ \\
\hline Médicos & 90 & 57,7 \\
Pediatras & 36 & 40,0 \\
Ginecologistas-obstetras & 17 & 18,9 \\
Clínicos gerais & 37 & 41,1 \\
Enfermeiros & 66 & 42,3 \\
Total & 156 & 100,0 \\
\hline
\end{tabular}

$36,5 \%$ dos respondentes; para $1,9 \%$ o distúrbio não pode ser evitado e $5,1 \%$ consideraram que todos os casos podem ser evitados, não sendo observada diferença significativa entre os médi$\cos \left(\right.$ as) e enfermeiros(as) $\left(\chi^{2}=2,560 ; p=0,764\right)$.

A Tabela 3 mostra a porcentagem dos profissionais de saúde que se sentem inseguros para orientar sobre o efeito teratogênico de alguns agentes. A Tabela 4 apresenta a porcentagem dos profissionais de saúde que referiram insegurança para orientar sobre a indicação e os riscos das técnicas utilizadas para o diagnóstico pré-natal, e a Tabela 5 , a porcentagem dos que responderam desconhecer os testes que fazem parte do Programa Nacional de Triagem Neonatal (PNTN) 6.

O tempo de formado dos profissionais de saúde influenciou somente na segurança para orientar sobre a biopsia de vilo corial. Os profissionais graduados há mais de 15 anos demonstraram maior segurança que os demais $\left(\chi^{2}=17,657 ; p=0,014\right)$.

A comparação das respostas dos médicos demonstrou diferença significativa somente em relação às técnicas de diagnóstico pré-natal. Como esperado, os ginecologistas referiram maior segurança que os demais para orientar sobre amniocentese $\left(\chi^{2}=7,045 ; \mathrm{p}=0,030\right)$, biópsia de vilo corial $\left(\chi^{2}=24,609 ; \mathrm{p}<0,001\right)$, cordocentese $\left(\chi^{2}=21,527 ; \mathrm{p}<0,001\right)$, translucência nucal $\left(\chi^{2}=6,869 ; \mathrm{p}=0,032\right)$ e teste triplo $\left(\chi^{2}=15,786 ; p<0,001\right)$

\section{Discussão}

O potencial de prevenção da deficiência mental aumentou muito nas últimas décadas 7,8,9, e, no Brasil, a implantação de programas preventivos é beneficiada pelo modelo de atenção à saúde pública vigente 10,11.

Em Maringá, não existe programa voltado à prevenção da deficiência mental e/ou defeitos congênitos, mas existem ações que fazem parte do protocolo de assistência à gestante e ao recém-nascido que procuram identificar fatores de risco associados a essa condição 12,13.

Mesmo assim, 75\% dos profissionais de saúde não foram capazes de assinalar a alternativa correta sobre a prevalência da deficiência mental e somente $11 \%$ responderam corretamente sobre a contribuição do genoma para a etiologia dessa condição. Para $2 \%$, a deficiência mental não pode ser evitada e $37 \%$ responderam não saber se existe prevenção, desconhecimento esse refletido na percentagem considerável de médicos e enfermeiros que responderam não saber se os testes para fenilcetonúria e hi- 
Características dos profissionais de saúde.

\begin{tabular}{|c|c|c|c|}
\hline & $\begin{array}{l}\text { Profissionais da } \\
\text { área médica }(n)\end{array}$ & $\begin{array}{l}\text { Profissionais da área } \\
\text { de enfermagem ( } n)\end{array}$ & Total (n) \\
\hline Gênero masculino & $54,2 \%(45)$ & $7,6 \%(5)$ & $33,6 \%(50)$ \\
\hline Média da idade (anos) & $41,3(d p=9,65)$ & $34,5(\mathrm{dp}=6,32)$ & $38,3(\mathrm{dp}=8,98)$ \\
\hline Tempo de formado (média em anos) & $16,5(d p=9,27)$ & $09,8(d p=6,21)$ & $13,7(\mathrm{dp}=8,71)$ \\
\hline Trabalham exclusivamente na rede pública & $27,0 \%(24)$ & $82,8 \%(53)$ & $\begin{array}{c}50,3 \%(77) \\
{\left[\chi^{2}=77,891 ; p<0,001\right]}\end{array}$ \\
\hline Tiveram disciplina de genética na graduação & $78,9 \%(71)$ & $90,9 \%(60)$ & $\begin{array}{c}84,0 \%(131) \\
{\left[\chi^{2}=4,08 ; p=0,043\right]}\end{array}$ \\
\hline Tiveram treinamento em genética médica & $5,6 \%(5)$ & $1,5 \%(1)$ & $\begin{array}{c}3,8 \%(6) \\
{\left[\chi^{2}=1,681 ; p=0,195\right]}\end{array}$ \\
\hline Lêem temas de genética médica & $42,7 \%(38)$ & $32,3 \%(21)$ & $\begin{array}{c}38,3 \%(54) \\
{\left[\chi^{2}=1,715 ; p=0,190\right]}\end{array}$ \\
\hline Raramente & $94,7 \%(36)$ & $100,0 \%(21)$ & $\begin{array}{c}96,4 \%(57) \\
{\left[\chi^{2}=0,982 ; p=0,322\right]}\end{array}$ \\
\hline Procedência científica & $86,7 \%(26)$ & $11,8 \%(2)$ & $\begin{array}{c}59,6 \%(28) \\
{\left[\chi^{2}=25,278 ; p<0,001\right]}\end{array}$ \\
\hline
\end{tabular}

Tabela 3

Porcentagem dos profissionais de saúde que referiram insegurança para orientar sobre o efeito teratogênico de alguns agentes.

\begin{tabular}{lccccccc}
\hline Alternativas & \multicolumn{2}{c}{ Médicos } & \multicolumn{2}{c}{ Enfermeiros } & \multicolumn{2}{c}{ Total } & Teste $\chi^{2}(p)$ \\
& $n$ & $\%$ & $n$ & $\%$ & $n$ & $\%$ & \\
\hline Hidantoína & 35 & 48,6 & 41 & 75,9 & 76 & 60,3 & $9,619(0,002)$ \\
Cocaína & 19 & 22,6 & 17 & 27,9 & 36 & 24,8 & $0,522(0,470)$ \\
Misoprostol & 39 & 51,3 & 41 & 85,4 & 80 & 64,5 & $14,944(0,001)$ \\
Etanol & 13 & 16,5 & 26 & 45,6 & 39 & 28,7 & $13,764(0,001)$ \\
Citomegalovirose & 6 & 7,1 & 17 & 28,8 & 23 & 16,0 & $12,281(0,001)$ \\
Diabetes mellitus & 22 & 28,9 & 12 & 22,2 & 34 & 26,2 & $0,739(0,390)$ \\
\hline
\end{tabular}

Tabela 4

Porcentagem dos profissionais de saúde que se sentem inseguros para orientar sobre a indicação e os riscos das técnicas utilizadas para diagnóstico pré-natal.

\begin{tabular}{lccccccc}
\hline \multirow{2}{*}{ Alternativas } & \multicolumn{2}{c}{ Médicos } & \multicolumn{2}{c}{ Enfermeiros } & \multicolumn{2}{c}{ Total } & \multicolumn{2}{c}{ Teste $\chi^{2}(p)$} \\
& $n$ & $\%$ & $n$ & $\%$ & $n$ & $\%$ & \\
\hline Amniocentese & 22 & 26,2 & 28 & 48,3 & 50 & 35,2 & $7,336(0,007)$ \\
Biopsia de vilo corial & 49 & 62,0 & 50 & 90,9 & 99 & 73,9 & $14,018(0,001)$ \\
Cordocentese & 49 & 64,5 & 44 & 78,6 & 93 & 70,5 & $3,078(0,079)$ \\
Translucência nucal & 37 & 45,7 & 44 & 77,2 & 81 & 58,7 & $13,705(0,001)$ \\
Teste triplo & 63 & 86,3 & 53 & 96,4 & 116 & 90,6 & $3,378(0,053)$ \\
\hline
\end{tabular}


Porcentagem dos profissionais de saúde que desconhecem os testes que fazem parte do Programa Nacional de Triagem Neonatal.

\begin{tabular}{lrrrrrrr}
\hline Testes & \multicolumn{2}{c}{ Médicos } & \multicolumn{2}{c}{ Enfermeiros } & \multicolumn{2}{c}{ Total } & Teste $\chi^{2}(p)$ \\
& \multicolumn{1}{c}{$\mathrm{n}$} & $\%$ & $\mathrm{n}$ & $\%$ & $\mathrm{n}$ & $\%$ & \\
\hline Doenças falciformes & 21 & 29,2 & 32 & 54,2 & 53 & 40,5 & $9,393(0,009)$ \\
Fenilcetonúria & 6 & 6,9 & 12 & 18,2 & 18 & 11,7 & $5,367(0,068)$ \\
Fibrose cística & 20 & 26,7 & 41 & 70,7 & 61 & 45,9 & $25,606(0,001)$ \\
Hipotireoidismo & 7 & 8,3 & 21 & 35,0 & 28 & 19,4 & $16,359(0,001)$ \\
\hline
\end{tabular}

potireoidismo estão incluídos no PNTN (8\% e $18,2 \% ; 9,5 \%$ e $35 \%$, respectivamente), apesar de esses fazerem parte oficialmente da triagem neonatal desde 1992.

Número relativamente elevado referiu insegurança para orientar sobre os efeitos teratogênicos de drogas e doenças de prevalência elevada, como o álcool, a citomegalovirose e o diabetes mellitus 14,15. A maioria também se sente insegura para orientar sobre as indicações e os riscos das técnicas de diagnóstico pré-natal, exceto em relação à amniocentese.

Não foi objetivo da pesquisa a avaliação aprofundada do conhecimento dos profissionais de saúde; porém, os dados obtidos demonstram que os participantes têm baixa percepção da relevância da deficiência mental para a morbidade da população e necessitam de maiores informações sobre os aspectos genéticos e ambientais relacionados a tal condição.

Ainda que inexistam na literatura nacional estudos semelhantes, impedindo a generalização e comparação dos dados, algumas pesquisas 16,17 têm demonstrado a falta de conhecimento dos profissionais de saúde sobre assuntos que envolvem noções de genética. Mesmo que as causas ambientais ainda sejam relevantes na etiologia da deficiência mental $2,7,8$, a necessidade de diagnóstico diferencial com doenças genéticas exige, dos profissionais de saúde, conhecimentos básicos nessa área da medicina.

Isso se justifica pelo fato de que muitas crianças com atraso global do desenvolvimento ou deficiência mental têm o diagnóstico e a terapia retardados pela dificuldade de encaminhamento a centros especializados 18,19. A situação é mais evidente nos casos de inaptidão leve ou moderada (QI entre 70 e 35). Embora de prevalência elevada, pois atinge entre 2 e $3 \%$ da população, o problema geralmente só é percebido entre os 7 e 10 anos, quando se observa que a criança não consegue desenvolver as habilidades próprias da idade e acompanhar o conteúdo escolar 20,21.

Pediatras, ginecologistas-obstetras, clínicos gerais e médicos do PSF, ou ainda enfermeiros, por manterem contato mais freqüente e precoce com esses pacientes, passam a ter uma grande responsabilidade no manejo do caso. Estando bem preparados para identificar os fatores de risco, conduzir eficientemente a investigação inicial e fazer o encaminhamento correto para os especialistas, poderão se constituir em um poderoso instrumento de prevenção da deficiência mental.

Promover ações de prevenção da deficiência mental em nível de baixa complexidade é necessário e possível, entretanto é preciso investir em pesquisas de avaliação do conhecimento e da atitude preventiva dos profissionais de saúde 22, e dos próprios usuários 23 , a fim de conhecer as deficiências e necessidades dessas populações, possibilitando que os recursos possam ser concentrados em programas exeqüíveis e com maior probabilidade de êxito. 


\section{Resumo}

O objetivo deste artigo foi averiguar o perfil do atendimento preventivo em deficiência mental na rede básica de saúde pública do Município de Maringá, Paraná, Brasil. A amostra foi composta por 90 médicos(as) das áreas de Ginecologia e Obstetrícia, Pediatria, Clínica Geral, Programa Saúde da Família e 66 enfermeiros(as). Foi aplicado questionário, por autopreenchimento, com respostas de múltipla escolha, entre agosto e dezembro de 2003. As variáveis qualitativas foram comparadas pelo teste do qui-quadrado, com nível de significância de 5\%. São apresentados dados parciais relacionados à percepção e ao conhecimento de profissionais de saúde sobre deficiência mental: $75 \%$ não foram capazes de assinalar a alternativa correta sobre a prevalência; $25 \%$ não sabem qual a contribuição do genoma para a etiologia; $37 \%$ não sabem se existe prevenção para a deficiência mental; $28 \%$ não se sentem seguros para orientar sobre o efeito teratogênico do etanol; 35\% referiram insegurança para orientar sobre amniocentese. Os dados demonstraram que os participantes têm baixa percepção da relevância da deficiência mental para a morbidade da população e necessitam de maiores informações sobre os aspectos genéticos e ambientais relacionados a tal condição.

Saúde Mental; Serviços de Saúde; Percepção

\section{Referências}

1. Curry CJ, Stevenson RE, Aughton D, Byrne J, Carey JC, Cassidy S, et al. Evaluation of mental retardation: recommendations of a consensus conference. Am J Med Genet 1997; 72:468-77.

2. Yeargin-Allsopp M, Murphy CC, Cordero JF, Decouflé P, Hallowell JG. Reported biomedical causes and associated medical conditions for mental retardation among 10-year-old children, metropolitan Atlanta, 1985 to 1987. Dev Med Child Neurol 1997; 39:142-9.

3. Instituto Brasileiro de Geografia e Estatística. Censo Demográfico 2000. http:/ / www.ibge.gov. $\mathrm{br} /$ home/estatistica/populacao/censo2000/popu lacao/censo2000_populacao.pdf (acessado em 20/Mai/05)

4. Enumo SRF, Kerbauy RR. Prevenção da deficiência mental: sua importância e concepções. Bol Psicol 1994; XLIV: 31-40.

5. Gil AC. Métodos e técnicas de pesquisa social. São Paulo: Editora Atlas; 1999.

6. Portaria n. 822/GM de 06 de junho de 2001. Institui, no âmbito do Sistema Único de Saúde, o Programa Nacional de Triagem Neonatal. http:// dtr2001.saude.gov.br/sas/PORTARIAS/Port2001/ GM/GM-822.htm (acessado em 20/Mai/2005).

7. Stevenson RE, Massey PS, Schroer RJ, McDermott S, Richter B. Preventable fraction of mental retardation: analysis based on individuals with severe mental retardation. Ment Retard 1996; 34:182-8.

\section{Colaboradores}

A. M. S. M. Moraes participou da idealização e desenvolvimento da pesquisa e redação do artigo. L. A. Magna co-orientou a pesquisa e colaborou na análise estatística. A. P. Marques-de-Faria orientou a pesquisa e contribuiu na redação e discussão do artigo.

\section{Agradecimentos}

À Coordenação de Aperfeiçoamento de Pessoal de Nível Superior (CAPES), Ministério da Educação, à Universidade Estadual de Maringá (UEM), aos profissionais de saúde da rede pública de saúde do Município de Maringá que participaram da pesquisa.
8. Calderón-González R, Calderón-Sepúlveda RE. Prevención del retraso mental. Rev Neurol 2003; 36:184-94.

9. World Health Organization. Mental and neurological disorders. http://www.who.int/mediacentre/factsheets/fs265/en/print.html (acessado em 17/Mai/2005).

10. Brasil. Norma operacional básica do Sistema Único de Saúde. Brasília: Diário Oficial da União 1996; 6 nov.

11. Andrade SM, Soares DA, Cordoni Jr. L. Bases da saúde coletiva. Londrina: Editora Eduel; 2001.

12. Chapman DA, Scott KG, Mason CA. Early risk factors for mental retardation: role of maternal age and maternal education. Am J Ment Retard 2002; 107:46-59.

13. Jelliffe-Pawlowski LL, Shaw GM, Nelson V, Harris JA. Risk of mental retardation among children born with birth defects. Arch Pediatr Adolesc Med 2003; 157:545-50.

14. Sanseverino MTV, Spritzer DT, Schüler-Faccini L, organizadores. Manual de teratogênese. Porto Alegre: Editora UFRGS; 2001.

15. Marcondes E, Vaz FAC, Ramos JLA, Okay Y, organizadores. Pediatria básica: pediatria geral e neonatal. São Paulo: Editora Sarvier; 2003.

16. Rim PHH, Magna LA, Ramalho, AS. Genética e prevenção da cegueira: percepção e conduta de oftalmologistas brasileiros. http://www.abonet. 
com.br/abo/664s/painel03.htm (acessado em 20/Mai/05).

17. Baars MJ, Scherpbier AJ, Schuwirth LW, Henneman L, Beemer FA, Cobben JM, et al. Deficient knowledge of genetics relevant for daily practice among medical students nearing graduation. Genet Med 2005; 7:295-301.

18. Penchaszadeh VB, Beiguelman B. Medical genetic services in Latin America: report of a meeting of experts. Rev Panam Salud Pública 1998; 3:409-20.

19. Marques-de-Faria AP, Ferraz VEF, Acosta AX, Brunoni D. Clinical genetics in developing countries: the case of Brazil. Community Genet 2004; 7:95-105.

20. Vasconcelos MM. Retardo mental. J Pediatr (Rio J) 2004; 80 (2 Suppl):71-82.
21. Tramontina S, Martins S, Michalowski MB, Ketzer CR, Eizirik M, Biederman J, et al. Estimated mental retardation and school dropout in a sample of students from state public schools in Porto Alegre, Brazil. Rev Bras Psiquiatr 2002; 24:177-81.

22. Enumo SRF, Trindade ZA. Ações de prevenção da deficiência mental, dirigidas a gestantes e recémnascidos, no âmbito da saúde pública da grande Vitória, ES. Psicol USP 2002;13:107-32.

23. Garcias LG, Schüler-Faccini L. The beliefs of mothers in southern Brazil regarding risk-factors associated with congenital abnormalities. Genet Mol Biol 2004; 27:147-53.

Recebido em 04/Jul/2005

Versão final reapresentada em 31/Out/2005

Aprovado em 26/Dez/2005 